\title{
Comparing Models GRM, Refraction Tomography and Neural Network to Analyze Shallow Landslide
}

\author{
Armstrong F. Sompotan ${ }^{1}$, Linus A. Pasasa ${ }^{2} \&$ Rachmat Sule $^{3}$ \\ ${ }^{1}$ Department of Physics, Manado State University, Tondano, 95618, Indonesia \\ ${ }^{2}$ Physics of Complex System, Institute of Technology Bandung, Bandung, Indonesia \\ ${ }^{3}$ Department of Geophysical Engineering, Institute of Technology Bandung, \\ Bandung, Indonesia \\ E-mail: armstrong.itb@gmail.com
}

\begin{abstract}
Detailed investigations of landslides are essential to understand fundamental landslide mechanisms. Seismic refraction method has been proven as a useful geophysical tool for investigating shallow landslides. The objective of this study is to introduce a new workflow using neural network in analyzing seismic refraction data and to compare the result with some methods; that are general reciprocal method (GRM) and refraction tomography. The GRM is effective when the velocity structure is relatively simple and refractors are gently dipping. Refraction tomography is capable of modeling the complex velocity structures of landslides. Neural network is found to be more potential in application especially in time consuming and complicated numerical methods. Neural network seem to have the ability to establish a relationship between an input and output space for mapping seismic velocity. Therefore, we made a preliminary attempt to evaluate the applicability of neural network to determine velocity and elevation of subsurface synthetic models corresponding to arrival times. The training and testing process of the neural network is successfully accomplished using the synthetic data. Furthermore, we evaluated the neural network using observed data. The result of the evaluation indicates that the neural network can compute velocity and elevation corresponding to arrival times. The similarity of those models shows the success of neural network as a new alternative in seismic refraction data interpretation.
\end{abstract}

Keywords: GRM; neural network; refraction tomography; shallow landslide.

\section{$1 \quad$ Introduction}

Landslide is one of the most costly and damaging natural hazards in Indonesia. As the case of shallow landslide at Kertasari Bandung that has slope with high permeable soil on top of low permeable bottom soil [1]. The low permeable bottom soil traps the water in the shallower, high permeable soil creating high water pressure in the top soil. As the top soil is filled with water and becomes heavy, slope becomes very unstable and slides over the low permeable bottom soil. Detailed investigations of landslides are essential to understand fundamental landslide mechanisms. Seismic refraction is a technique that has

Received September $28^{\text {th }}, 2009$, Revised April $2^{\text {nd }}, 2011$, Accepted for publication May $2^{\text {nd }}, 2011$.

Copyright (C) 2011 Published by LPPM ITB \& PII, ISSN: 1978-3051, DOI: 10.5614/itbj.eng.sci.2011.43.3.1 
been used to investigate landslides since the early 1960's [2]. The velocity structure of a landslide mass, the depth to the sliding surface, and the lateral extent of a landslide are variables that may be estimated using seismic refraction. The basis of the interpretations is the difference in the physical properties of the sliding materials and the underlying undisturbed sediments that result in different seismic velocities [2].

Conventional seismic refraction data have been processed and presented utilizing a number of methods for a very long time. Palmer's approach using the generalized reciprocal method (GRM) has been the industry standard for assessing a layered earth using first arrival times of body wave energy to produce images of the subsurface. GRM is a technique for delineating undulating refractors at any depth from in-line seismic refraction data consisting of forward and reverse travel times [3]. GRM can be used to model velocity structures of some landslides. These methods are most applicable to sites where subsurface layers dip less than approximately $20 \mathrm{o}$ and have nearly uniform velocities, for these methods assume a layered model and continuity of refractor surfaces across a profile. However, the velocity structures of landslides can be complex, making them difficult to accurately model using GRM. Refraction tomography, another method of interpreting seismic refraction data, is capable of modeling these complex velocity structures. These methods, more complex mathematical approaches, all termed tomography, vary to some degree in their analysis, but the image results are generally comparable [4]. Inversion modeling often requires a lot of time and using complicated methods. In contrast to time consuming and complicated numerical methods, neural network is found to be of potential applicability $[5,6]$. The trained neural network indicates that the neural network can satisfactorily compute seismic reflection velocity and dips corresponding to travel-times [6]. In this study, neural network ability to establish a relationship between an input and output space is considered to be appropriate for mapping seismic refraction velocity and elevation corresponding to arrival times. The purpose of this paper is to introduce a new workflow in analyzing seismic refraction data.

\section{Research Method}

The methodology of the seismic refraction analysis consists two parts; there are field measurement part and data processing part. The seismic refraction survey was able to carry out using the seismograph with acquisition unit of the highspeed interface system. In this study, the seismic refraction testing was located in landslide area at the Kertasari Bandung. The Kertasari landslide was selected because of its shallow sliding surface at a depth of about $5 \mathrm{~m}$. The spread line employed was $69 \mathrm{~m}$ based on $3 \mathrm{~m}$ geophone spacing due to the constraint of the site. The layout of the seismic refraction set up is schematically shown in Figure 
1. The 24 geophones are placed on the linear imaginary line and the seismograph box is set to connect the 12th and 13th geophone. The several shot points of the $10 \mathrm{~kg}$ wood hammer in order to generate the $\mathrm{P}$ waves are located at the 1 st to 7 th point in the spread line.

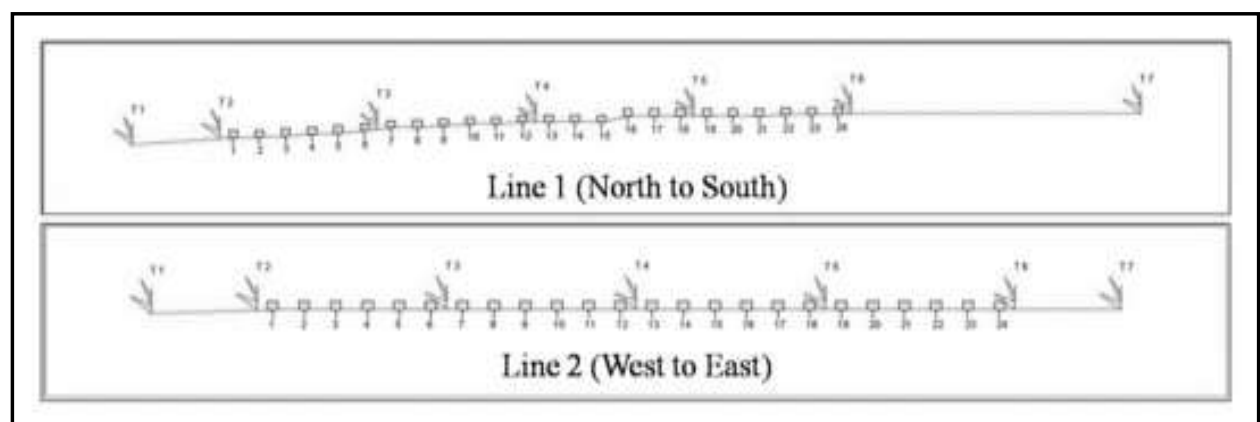

Figure 1 The seismic refraction set up. The spread line employed was $69 \mathrm{~m}$ based on $3 \mathrm{~m}$ geophone spacing due to the constraint of Kertasari site. The 24 geophones are placed on the line (two lines with two different directions) and the seismograph box is set to connect the 12th and 13th geophone. Several shot points are located at the 1 st to 7 th point in the spread line.

The data processing technique of the seismic refraction method is explained schematically in Figure 2. The analogue data of the seismic wave propagation is directly resulted from the field measurement equipment. The analogue data is then transferred into the digital format data. The important information of the digital data for the seismic refraction method is the first arrival time information of $\mathrm{P}$-waves which propagates to the geophones. To invert the data, three methods were used; GRM, refraction tomography and neural network (as a new alternative).

The GRM is a seismic inversion method that uses the refracted arrival Traveltime data for both forward and reverse shots, and the reciprocal time [3, $7,8]$. The GRM is able to provide good estimates of the seismic velocities within the refractor, and the structure or shape of the refracting interface [7]. The major difference between the GRM and other seismic refraction inversion methods is that the former applies a variable refraction migration which can be often be useful in resolving undetected layers, variable velocity media and anisotropy under favorable conditions [9]. With the GRM, a range of offset distances is computed, usually from zero to a value in excess of the estimated true value. The optimum value, for which the refractor velocity analysis function displays a minimum of artifacts caused by the irregular refractor interface, is selected by inspection of the graphs. The GRM consists of two algorithms: the velocity analysis function from which the refractor velocity is derived, and the time-depth function which is a measure of the depth to the 
refractor in units of time. The two functions are calculated for different values of the horizontal separation between forward and reverse receivers (XY values), with the optimum value being determined from inspection of the resulting functions. The GRM originated as a manual method but nowadays can be implemented with commercial software (e.g., WinSism by W-GeoSoft [10]).

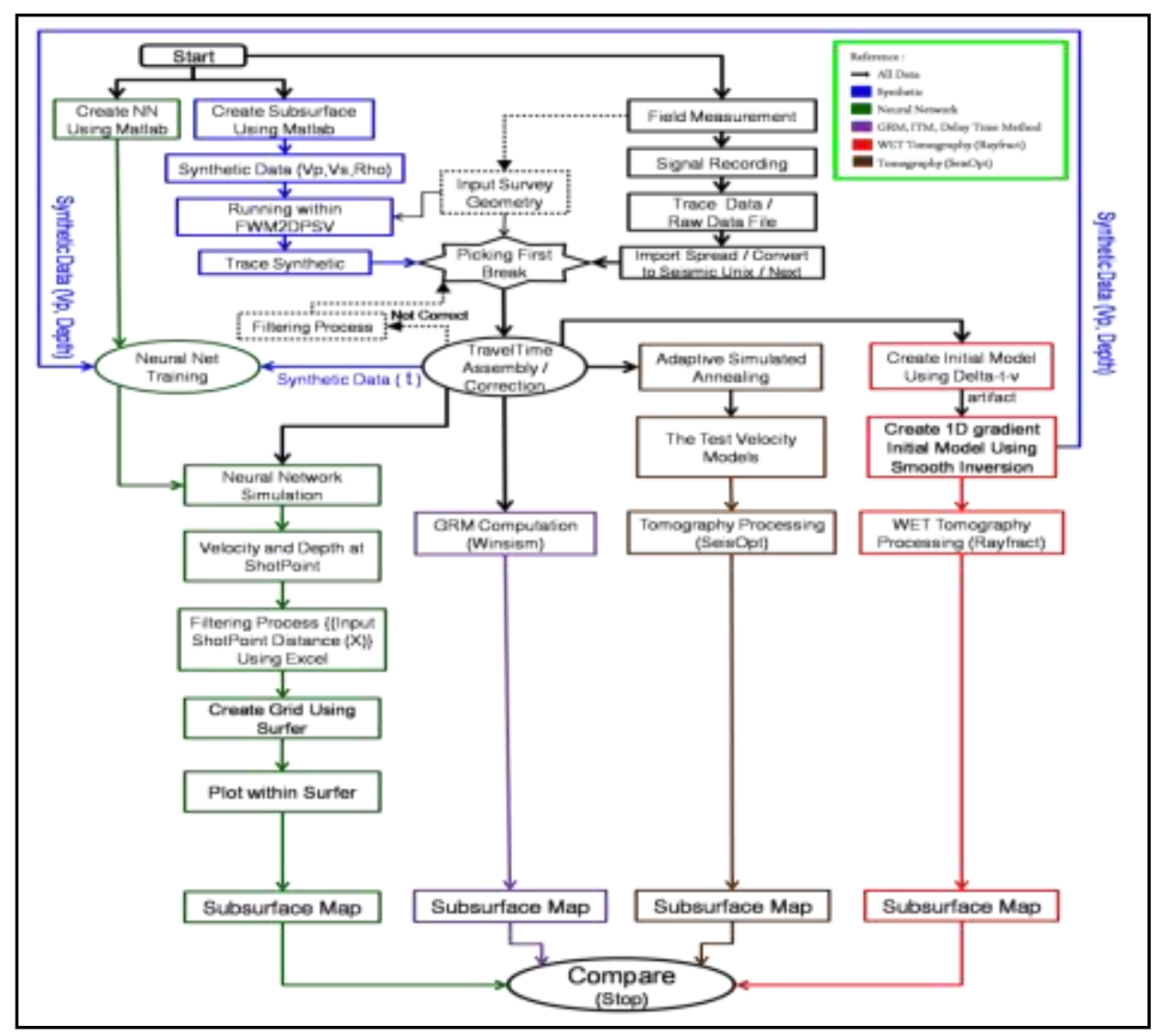

Figure 2 Research Schema. For the first, Neural Network was created and then we trained it with the synthetic data. The input is time of subsurface synthetic created using Matlab and then we run within FWM2DPSV. The Target Outputs are velocity and depth of the initial model of the travel time synthetic created using Rayfract ${ }^{\mathrm{TM}}$. After the training, we simulated the Neural Network with the field data. All of the systems used the same field measurement data. The outputs of the simulation are velocity and depth at shot point. It seems similar to the shot point depth computation or Intercept Time Method. After that, the data was filtered using Microsoft Excel, and then Grid using Surfer was created, and we plot them within Surfer to be a Subsurface Map that we compared it with the Subsurface Map of GRM, Rayfract ${ }^{\mathrm{TM}}$ Software and SeisOpt@2D Software. 
Two commercially-available refraction tomography software systems were used to produce $\mathrm{P}$ wave velocity tomograms. The two systems are Rayfract ${ }^{\mathrm{TM}}$ by Intelligent Resources, Inc [11] and SeisOpt@2D by Optim LLC [12]. Each of the systems contains three important components: a forward model for calculating source to receiver first arrival times based upon the current velocity model, an inversion routine for adjusting the velocity model until an acceptable match between calculated and measured first-arrival travel times is obtained, and a means for generating an initial velocity model [13].

Rayfract implements Wavepath Eikonal Traveltime tomography (WET) [14] with the Fresnel volume approach to inversion [15]. The WET inversion method is founded upon a backprojection formula for inverting velocities from travel times computed by a finite-difference solution to the Eikonal equation [16]. Rayfract provides two options for generating an initial model to start WET inversion: uses the Delta-t-V method included in Rayfract, or uses the "smooth inversion" algorithm that automatically creates a one dimensional model based on Delta-t- $\mathrm{V}$ a result that is then extended to cover the two-dimensional area [4]. The "smooth inversion" algorithm is intended to eliminate artifacts that can sometimes be produced by the Delta-t-V solutions.

SeisOpt@2D is based upon a Monte Carlo-based optimization scheme described by Pullammanappallil and Louie [17]. For forward model, a finitedifference solution of the Eikonal equation [18] computes first-arrival travel times through the velocity model. Inversion is accomplished via a generalized simulated annealing global optimization algorithm. Pullammanappallil and Louie [17] demonstrate that the simulated annealing inversion algorithm is independent of the initial model. The SeisOpt@2D user must specify the vertical and horizontal spatial resolution (cell size) of the model. By default, a constant velocity is assigned to the model by SeisOpt@2D to begin the optimization. Alternatively, the user can input results from a previous run as an initial model, or fully specify an initial velocity model.

The neural network has the ability to map seismic velocity. This study is based on application of feed-forward neural network. The neural network contains three layers that are an input layer, a hidden layer and an output layer (Figure $3)$. There is no connection between neurons in the same layer. Connections are only between adjacent layers [19]. A neuron, a simple processing node, is used to calculate the output $a$ according to an input $n$. The weighted (W) sum of all outputs of neurons in previous layers is the value of input $n$ for neuron $i$ outside of input layer. Neurons in previous layers are indicated by index $j$.

$$
n_{i}=b+\sum_{j} W_{i j} a_{j}
$$


Based on a tan-sigmoid function, the value of output is calculated as follows,

$$
a_{i}=\frac{2}{1+e^{-n_{i}}}-1
$$

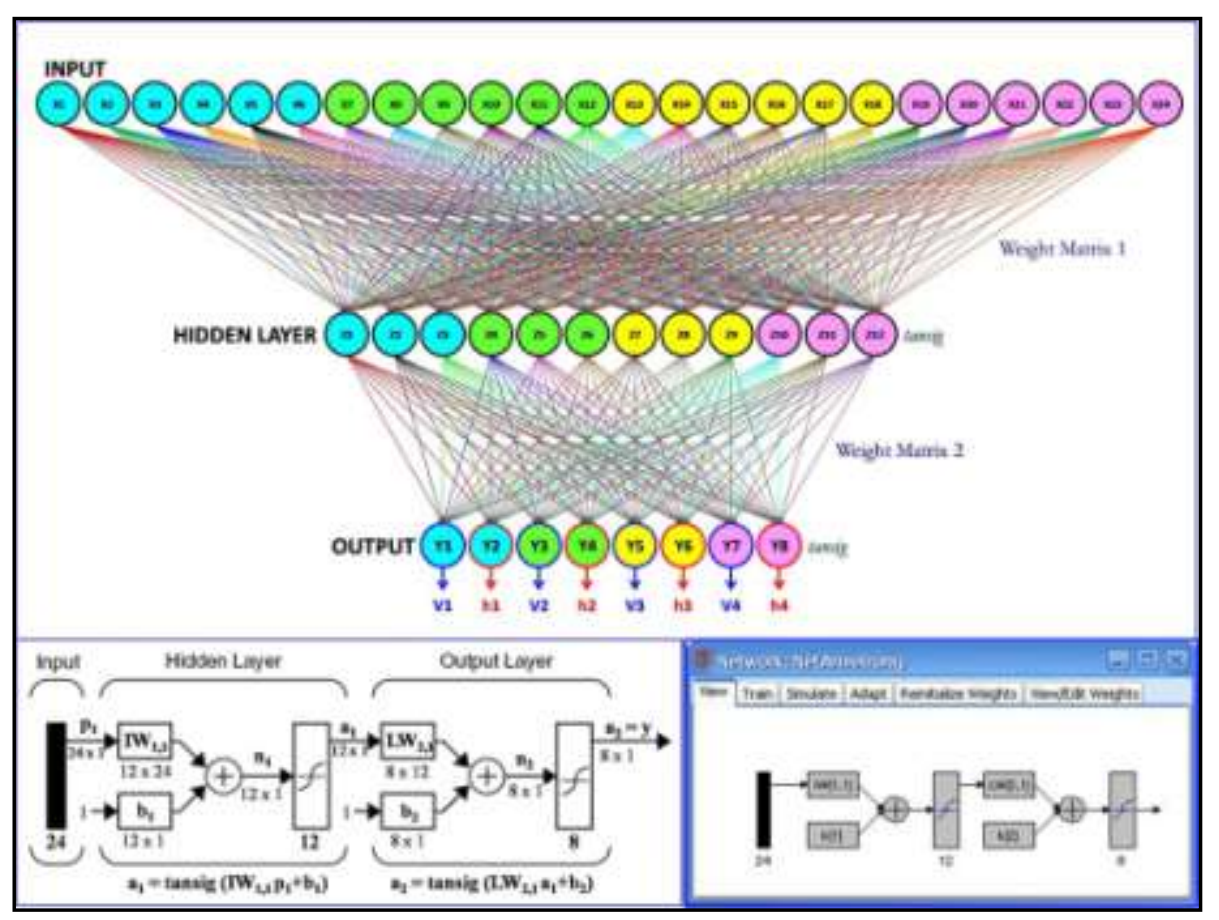

Figure 3 Neural Network Structure. The feed-forward back propagation neural network consists of three layers. The input has 24 neurons (6 traces for each layer), the output has 8 neurons (velocity and elevation for each 4 layers) and the hidden layer consists of 12 neurons. The number of weights are $(24 \mathrm{x} 12)+(12 \mathrm{x}$ $8)=384$. The information flows from input to hidden layer and then through output. Picked travel times were considered as inputs and the corresponding velocity and elevation as outputs. The generalization phase calculates the model characteristics (i.e., velocity and depth) corresponding to unknown input travel times. The performance goal for all neural network applications was set to 1e005. In other words, the generalization performance is considered accurate for different models, when this goal is achieved.

A training process is initiated in which the structure and output function remain unchanged. The process of initializing the weights $W$ comprises by training in order to minimize the error between the computed output and the desired output for all samples. In this study, picked travel times were considered as inputs and the corresponding velocity and depth as outputs. The overall procedure is shown in Figure 2. Corresponding travel times were calculated by a forward 
modeling scheme using FWM2DPSV program [20] (by the help Matlab software). The output of the forward modeling is a synthetic trace. Synthetic data are picked as input. First break data were also modeled with inversion method, where the velocity and depth of the initial model used as the target data. The corresponding data sets (i.e., arrival times as input and velocity and depth as output) were fed to neural network in order to train the networks. Afterwards the neural network was tested using parts excluded from the training set. This procedure or, generalization phase calculates the model characteristics (i.e., velocity and depth) corresponding to unknown input travel times.

The performance goal for all neural network applications was set to 1e-005. In other words, the generalization performance is considered accurate for different models, when this goal is achieved.

\section{$3 \quad$ Results and Discussions}

The interpretation of the seismic refraction profile provides a four layer model: The first layer shows a velocity of 343-449 m/s and corresponds to top soil. Its thickness varies from $1 \mathrm{~m}$ to $5 \mathrm{~m}$. The second layer shows velocity in the range of 449 to $837 \mathrm{~m} / \mathrm{s}$ (Depth 5-10m). It might be associated to smooth grain above the tuff layer. The third layer shows velocity in the range of 837 to $1100 \mathrm{~m} / \mathrm{s}$. It might be associated to tuff above the clay basement. The fourth layer shows clay stone. Basement with a velocity of $1100-1500 \mathrm{~m} / \mathrm{s}$ is located at a depth of about $23 \mathrm{~m}$ (Figure 5 and 6 ).

The analysis of neural network model provides a similar model. The principal approach in this method was considered to be the subsurface with the different velocity. For this geological model we calculated 700 data sets with different velocities, depths and dips. The profiles have been provided for these 700 examples with $3 \mathrm{~m}$ receiver separation for 24 geophone positions according to source location. The depths were determined based on input arrival time. The feed-forward backpropagation neural network consists of three layers. The input has 24 neurons ( 6 traces for each layer), the output has 8 neurons (velocity and elevation for each 4 layers) and the hidden layer consists of 12 neurons. In this experiment the number of weights or connections between neurons are (24 x 12) $+(12 \times 8)=384$.

The main problem in neural network training, which is done to produce a network that is capable to map the seismic velocity data based on input arrival time, is the determination of appropriate training targets. In other words it is very difficult or even impossible in that an equation consisting of three parameters that are closely interconnected is done an attempt to produce two unknown parameters based on the known parameter without external parameter 
assistance. Referring to the intercept-time method, the depth at every shot point can be calculated after discovery of the velocity and intercept time at every shot point. Both parameters are obtained through linear regression of travel time equation with the help of two known parameters of offset and arrival time. In other words, intercept time and velocity can be determined if the arrival time and offset are known. If the velocity and intercept time have known, the depth can be obtained. If applied in neural network training the arrival time and velocity are used to obtain intercept time with the help of the offset for determining the depth, so instead arrival time, offset and depth are used to determine the intercept time and velocity. Of understanding can be seen that the offset is very decisive role, but in this study did not use offset as an input so it will be very difficult for the neural network to determine the velocity and depth simultaneously. If one considered the same output for each layer, it will be very easy in training. The solution is to make an example. The depth is considered known, so the velocity can be determined easily based on arrival time. In this case, the arrival time was associated directly with the depth and neural network was trained to calculate the appropriate velocity. In this study, 24 input arrival times are divided into 4 layers, where 6 trace represent a layer. Each layer was represented by an arrival time. It is the average arrival time of six trace. $-1 / 2$ average arrival time in each layer is treated as a point of depth. The point of depth was later occupied by the velocity based on arrival time.

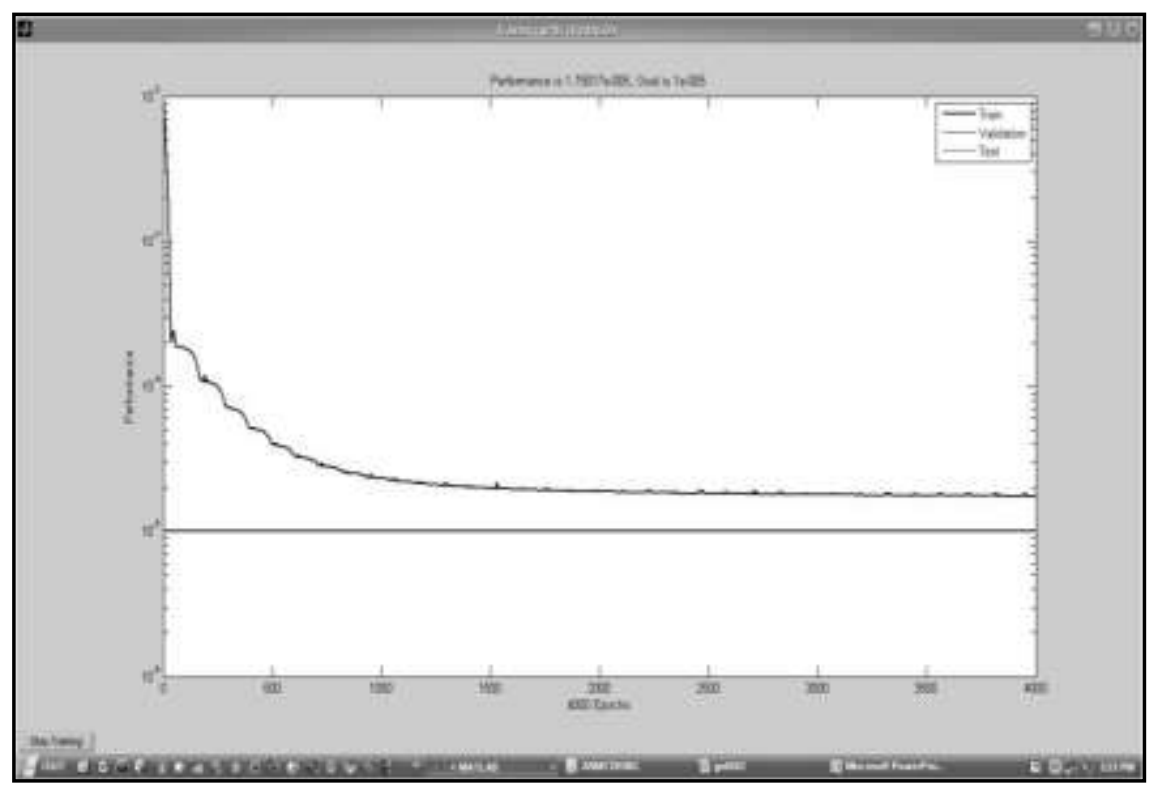

Figure 4 Screen captures of the neural network performance. The performance function of 0.0000175017 was achieved after 4,000 iterations that it wasn't close enough to the goal (1e-005) but it was enough for the evaluation. 
Set of arrival time in second and elevation in $\mathrm{km}$. The velocities were normalized to $10 \mathrm{~km} / \mathrm{s}$. After 4,000 iterations a performance function of 0.0000175017 was achieved that it wasn't close enough to the goal (1e005) as it can be seen in Figure 4.

The results of simulating the outputs for observed data are presented in Figure 5 and 6 . The velocity calculation showed relatively high accuracy. All models show the depth of the sliding surface (The Kertasari landslide) of about $5 \mathrm{~m}$.

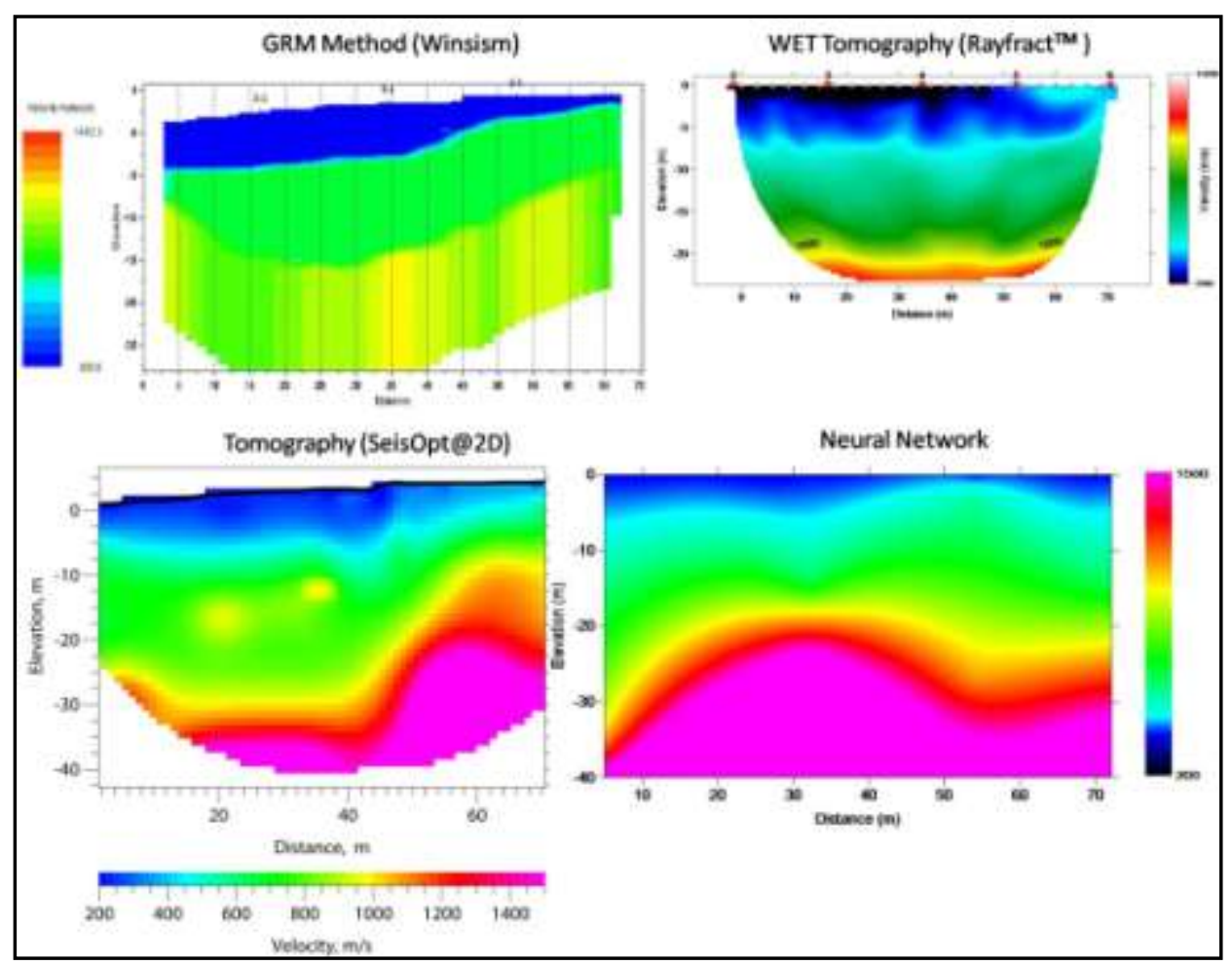

Figure 5 Comparing Models; Line 1. The results show that the velocities are similar. The profile provides a four layer model: The first layer shows the top soil with velocity of $343-449 \mathrm{~m} / \mathrm{s}$. The second layer shows the smooth grain above the tuff layer with velocity in the range of 449 to $837 \mathrm{~m} / \mathrm{s}$ (depth $5-10 \mathrm{~m}$ ). The third layer shows velocity in the range of 837 to $1100 \mathrm{~m} / \mathrm{s}$. It might be associated to tuff above the clay basement. The fourth layer shows basement with a velocity of $1100-1500 \mathrm{~m} / \mathrm{s}$ that is located at a depth of about $23 \mathrm{~m}$. 


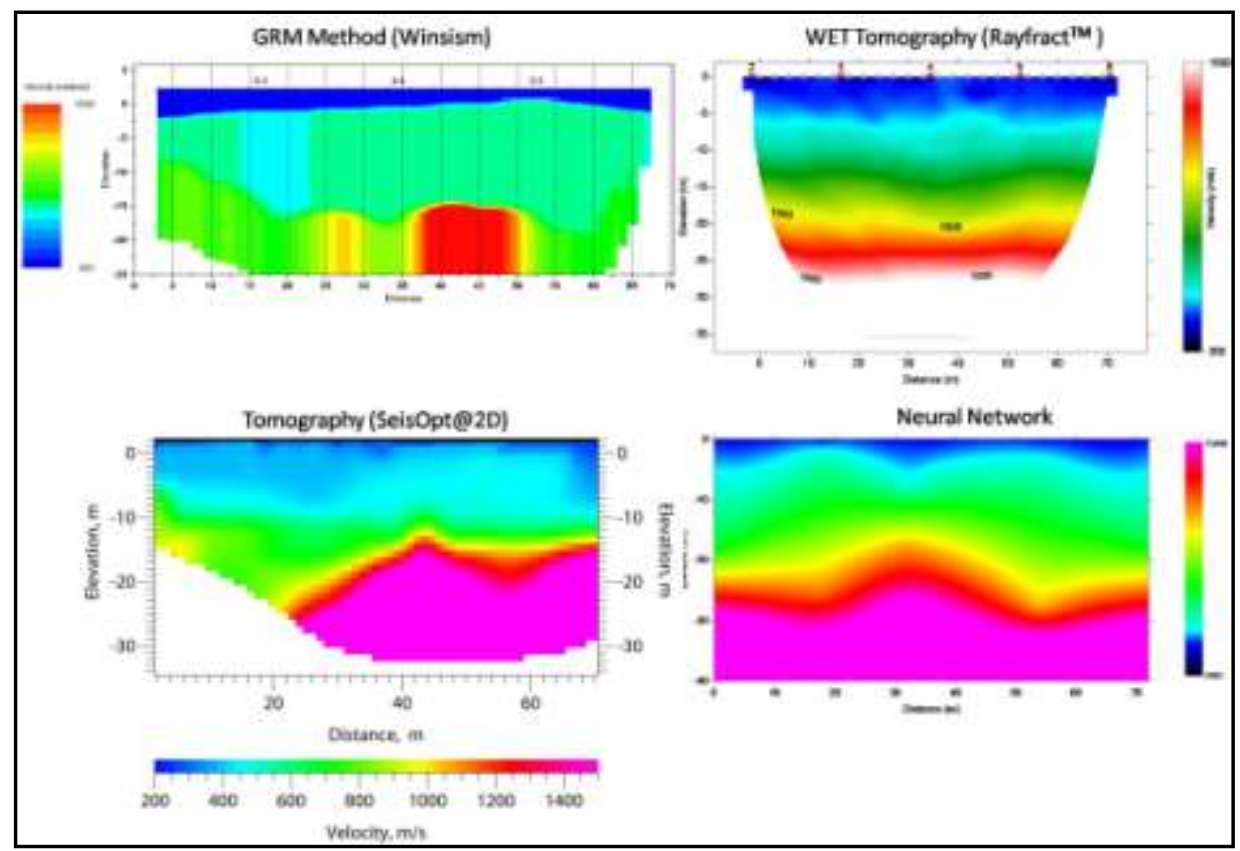

Figure 6 Comparing Models; Line 2. The velocities are similar with model line 1. However the results indicated that the velocity errors increase with increasing the depth of the layers.

\section{Conclusions}

All models show the depth of the sliding surface (The Kertasari landslide) of about $5 \mathrm{~m}$. The similarity of those models shows the success of neural network as a new alternative in seismic data interpretation. However the results of this study indicate that the velocity errors increase with the depth of the layers. Therefore, the approach for reducing the velocity error intervals requires further research.

\section{References}

[1] DESDM, Laporan Singkat Bencana Alam Gerakan Tanah di Kecamatan Kertasari Kabupaten Bandung Jawa Barat, Pusat Vulkanologi dan Mitigasi Bencana Geologi, Badan Geologi-Departemen Energi dan Sumber Daya Mineral, 2008.

[2] Narwold, C.F. \& Owen, W.P, Seismic Refraction Analysis of Landslides, Proceedings of the Geophysics 2002 Conference, Los Angeles, California, 2002.

[3] Palmer, D., An Introduction to The Generalized Reciprocal Method of Seismic Refraction Interpretation, Geophysics, 46, pp. 1508-1518, 1981. 
[4] Sheehan, J.R., Doll, W.E. \& Mandell, W.A., An Evaluation of Methods and Available Software for Seismic Refraction Tomography Analysis, Journal Environmental and Engineering Geophysics, 10, pp. 21-34, 2005.

[5] Baronian, C., Riahi, A. \& Lucas, C., Applicability of Artificial Neural Networks for Obtaining Velocity Models from Synthetic Seismic Data, International Journal of Earth Sciences (Geol Rundsch), 98, pp. 11731184, 2008.

[6] Baronian, C., Riahi, A., Lucas, C. \& Mokhtari, M., A Theoretical Approach to Applicability of Artificial Neural Networks For Seismic Velocity Analysis, Journal of Applied Sciences, 7, pp. 3659-3668, 2007.

[7] Palmer, D., A Simple Approach to 3D Shallow Refraction Seismology, ASEG 15th Geophysical Conference and Exhibition, Brisbane, 2001.

[8] Palmer, D., Nikrouz, R. \& Spyrou, A., Statics Corrections for Shallow Seismic Refraction Data, Exploration Geophysics, 36, pp. 7-17, 2005.

[9] Whiteley, R.J. \& Eccleston, P.J., Comparison of Shallow Seismic Refraction Interpretation Methods for Regolith Mapping, Proceedings of the 18th ASEG Geo Conference, 2006.

[10] Jenny, J., Borreguero, M. \& Burgisser, A., Winsism 10 Seismic Refraction Processing Software for Windows ${ }^{\circledR}$ : Instruction Manual, WGeosoft Geo2X, Geneva, 2008.

[11] Intelligent Resources, Seismic Refraction Data Interpretation with RAYFRACT TM Software, Rayfract tutorial, Intelligent Resources Inc., 2007.

[12] Optim, User's Manual SeisOpt ${ }^{\circledR} @ 2 D^{T M}$ Version 5.0, Optim Inc, Nevada, 2006.

[13] Hiltunen, D. R., Hudyma, N., Quigley, T. P. \& Samakur, C., Ground Proving Three Seismic Refraction Tomography Programs, Submitted for Presentation at 2007 Annual Meeting of Transportation Research Board, Washington D.C., 2007.

[14] Schuster, G.T. \& Quintus-Bosz, A., Wavepath Eikonal Traveltime Inversion: Theory, Geophysics, 58, pp. 1314-1323, 1993.

[15] Watanabe, T., Matsuoka, T. \& Ashida, A., Seismic Traveltime Tomography Using Fresnel Volume Approach, SEG, Expanded Abstracts 18, pp. 1402-1405, 1999.

[16] Qin, F., Luo, Y., Olsen, K.B., Cai, W. \& Schuster, G.T., FiniteDifference Solution of The Eikonal Equation Along Expanding Wavefront, Geophysics, 57, pp. 478-487, 1992.

[17] Pullammanappallil, S.K. \& Louie, J.N., A Generalized SimulatedAnnealing Optimization for Inversion of First Arrival Times, Bulletin of the Seismological Society of America, 84, pp. 1397-1409, 1994.

[18] Vidale, J.E., Finite-Difference Calculation of Traveltimes, Bulletin of the Seismological Society of America, 78, pp. 2062-2076, 1988. 
[19] Demuth, H., Beale, M. \& Hagan, M., Neural Network Toolbox ${ }^{T M} 6$ User's guide, MATLAB ${ }^{\circledR}$, The MathWorks, Inc., United States of America, 2008.

[20] Operto, S., Brossier, R. \& Virieux, J., Documentation of FWM2DPSV Program: 2D P-SV Finite-Difference Time-Domain Modelling of Elastic Wave Propagation, Technical report No. 7 - SEISCOPE Project, SEISCOPE Consortium, France, 2007. 